\title{
Delineation of the genetic and clinical spectrum of Phelan-McDermid syndrome caused by SHANK3 point mutations
}

Silvia De Rubeis ${ }^{1,2+} \mathbb{D}$, Paige M. Siper ${ }^{1,2+} \mathbb{D}$, Allison Durkin ${ }^{1} \mathbb{D}$, Jordana Weissman ${ }^{1} \mathbb{D}$, François Muratet ${ }^{1,2}$,

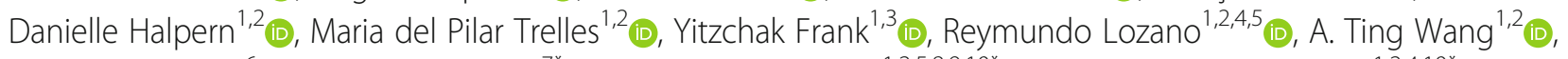
J. Lloyd Holder $\mathrm{rr}^{6} \mathbb{D}$, Catalina Betancur ${ }^{7^{*}} \mathbb{D}$, Joseph D. Buxbaum ${ }^{1,2,5,8,9,10^{*}} \mathbb{D}$ and Alexander Kolevzon ${ }^{1,2,4,10^{*}}$ (D)

\begin{abstract}
Background: Phelan-McDermid syndrome (PMS) is a neurodevelopmental disorder characterized by psychiatric and neurological features. Most reported cases are caused by 22q13.3 deletions, leading to SHANK3 haploinsufficiency, but also usually encompassing many other genes. While the number of point mutations identified in SHANK3 has increased in recent years due to large-scale sequencing studies, systematic studies describing the phenotype of individuals harboring such mutations are lacking.

Methods: We provide detailed clinical and genetic data on 17 individuals carrying mutations in SHANK3. We also review 60 previously reported patients with pathogenic or likely pathogenic SHANK3 variants, often lacking detailed phenotypic information.

Results: SHANK3 mutations in our cohort and in previously reported cases were distributed throughout the protein; the majority were truncating and all were compatible with de novo inheritance. Despite substantial allelic heterogeneity, four variants were recurrent (p.Leu1 142Valfs*153, p.Ala1227Glyfs*69, p.Arg1255Leufs*25, and c.2265+1G>A), suggesting that these are hotspots for de novo mutations. All individuals studied had intellectual disability, and autism spectrum disorder was prevalent (73\%). Severe speech deficits were common, but in contrast to individuals with 22q13.3 deletions, the majority developed single words, including $41 \%$ with at least phrase speech. Other common findings were consistent with reports among individuals with 22q13.3 deletions, including hypotonia, motor skill deficits, regression, seizures, brain abnormalities, mild dysmorphic features, and feeding and gastrointestinal problems.
\end{abstract}

Conclusions: Haploinsufficiency of SHANK3 resulting from point mutations is sufficient to cause a broad range of features associated with PMS. Our findings expand the molecular and phenotypic spectrum of PMS caused by SHANK3 point mutations and suggest that, in general, speech impairment and motor deficits are more severe in the case of deletions. In contrast, renal abnormalities associated with 22q13.3 deletions do not appear to be related to the loss of SHANK3.

Keywords: SHANK3, Phelan-McDermid syndrome, 22q13 deletion syndrome, Sequence variants, Phenotype, Autism spectrum disorder, Intellectual disability

\footnotetext{
* Correspondence: catalina.betancur@inserm.fr; joseph.buxbaum@mssm.edu; alexander.kolevzon@mssm.edu

Catalina Betancur, Joseph D. Buxbaum, and Alexander Kolevzon are co-senior authors.

${ }^{\dagger}$ Equal contributors

${ }^{7}$ Sorbonne Université, INSERM, CNRS, Neuroscience Paris Seine, Institut de

Biologie Paris Seine, 75005 Paris, France

${ }^{1}$ Seaver Autism Center, Icahn School of Medicine at Mount Sinai, New York, NY 10029, USA

Full list of author information is available at the end of the article
} 


\section{Background}

Phelan-McDermid syndrome (PMS, OMIM 606232) is a rare neurodevelopmental disorder characterized by neonatal hypotonia, global developmental delay, intellectual disability (ID), severely delayed or absent speech, and frequent autism spectrum disorder (ASD) [1]. The neurobehavioral phenotype of PMS is usually severe. In a prospective study of 32 PMS individuals, 77\% manifested severe-to-profound ID and $84 \%$ met criteria for ASD using gold standard diagnostic tools [2]. Dysmorphic features are usually mild and include long eyelashes, large or prominent ears, bulbous nose, pointed chin, fleshy hands, and dysplastic toenails [1]. Additional features include gastrointestinal problems, seizures, motor deficits, structural brain abnormalities, renal malformations, lymphedema, and recurrent infections [1].

The major neurodevelopmental features of PMS are caused by deletions or mutations of the SHANK3 gene, which encodes a scaffolding protein of the postsynaptic density of glutamatergic synapses. Most reported cases of PMS are caused by 22q13.3 deletions, which usually encompass many genes and can extend up to $9.2 \mathrm{Mb}$ [2-4]. Genotype-phenotype analyses indicate that the size of the deletion and the number and/or severity of clinical manifestations are positively correlated $[2,4-7]$. Specifically, correlations have been reported between deletion size and hypotonia [5-7], developmental delay [5-7], dysmorphic features [2, 7], speech abilities [4], social communication deficits related to ASD [2], and other medical conditions [2]. Furthermore, individuals with small terminal deletions may have more favorable developmental trajectories than those with larger deletions [8].

De novo truncating and missense mutations in SHANK3 have been identified in cohorts ascertained for ASD [9-16] or ID [17-21]. In addition, there is a single report of two families ascertained for schizophrenia with mutations in SHANK3; affected individuals also had ID [22]. Despite the increasing number of mutations in SHANK3, their prevalence in PMS and more broadly in ASD is underestimated because clinical sequencing is still uncommon compared to chromosomal microarray. In addition, SHANK3 has been poorly covered by whole exome sequencing due to high GC content $[13,23]$, and there is little in the PMS phenotype that would prompt a clinician to specifically target SHANK3 for optimized Sanger sequencing. We and others estimate that SHANK3 haploinsufficiency might account for up to $1 \%$ of more severely affected ASD cases [13, 23].

Given the dearth of identified cases with SHANK3 mutations, analyses of PMS cohorts have largely focused on individuals with 22q13.3 deletions [2-8, 24]. Only two studies on PMS have included a few individuals carrying SHANK3 mutations [2, 24]. These observations have been complemented by the description of a small number of individuals identified through SHANK3 targeted sequencing in ASD cohorts [9-13]. Large-scale sequencing studies have been instrumental in revealing additional SHANK3 mutations but have not provided detailed phenotypic information [14-16, 19-21].

The limited number of subjects with SHANK3 mutations examined thus far, and the lack of systematic clinical evaluation have hindered the characterization of the phenotypic spectrum associated with SHANK3 mutations. Here, we aimed to delineate the genetic spectrum of SHANK3 mutations and their associated phenotype in relationship to PMS features.

\section{Methods}

\section{Participants}

The study includes 14 participants (S1-S14) enrolled at the Seaver Autism Center for Research and Treatment at the Icahn School of Medicine at Mount Sinai, and three individuals (B1-B3) evaluated at Baylor College of Medicine. Individuals were referred through the PhelanMcDermid Syndrome Foundation, ongoing research studies, and communication between families. The study was approved by the Program for the Protection of Human Subjects at the Icahn School of Medicine at Mount Sinai and the Baylor College of Medicine Institutional Review Board. Parents or legal guardians provided informed consent for participation and publication. Consent was also obtained to publish the photos shown in Fig. 1.

\section{Genetic testing}

All mutations were identified and/or validated by Clinical Laboratory Improvement Amendments (CLIA)certified laboratories. The mutation in individual S1 was identified by whole exome sequencing (WES) and then validated by Sanger sequencing at the Seaver Autism Center [2] and by GeneDx. The mutation in S2 was identified by panel sequencing at the Michigan Medical Genetics Laboratories. The mutation in S3 was identified and validated at Seaver as previously reported [2] and further confirmed by Athena Diagnostics. The mutation in S4 was identified through clinical WES by the Columbia University Laboratory of Personalized Medicine. The mutations in S5, S11, and B1 were identified through clinical WES by the Medical Genetics Laboratory at the Baylor College of Medicine. The mutations in S6, S7, S9, S10, S12, and S14 were identified through clinical WES by GeneDx. The mutation in $\mathrm{S} 8$ was identified through clinical WES by AmbryGenetics. The variants in S13 were identified at the Seaver Autism Center and confirmed by GeneDx. The mutation in B2 and B3 was identified through clinical WES by Transgenomic.

Variants were described according to the Human Genome Variation Society guidelines. As reported previously 
[2], the human genome reference assembly (GRCh37/ hg19 and GRCh38/hg38) is missing the beginning of exon 11 (NM_033517.1:c.1305_1346, 5' -cccgagcgggcccg gcggccccggcccogcgeccggccccgg-3', coding for 436-PSG PGGPGPAPGPG-449). We numbered nucleotide and amino acid positions according to the SHANK3 RefSeq mRNA (NM_033517.1) and protein (NP_277052.1) sequence, in which this mistake has been corrected. Variants were interpreted according to the American College of Medical Genetics and Genomics (ACMG) guidelines [25].

\section{Review of previously reported SHANK3 mutations}

We searched the literature for pathogenic or likely pathogenic mutations in SHANK3 and retrieved the molecular and clinical information (Additional file 1: Tables S1-S3). We also included mutations reported in ClinVar (http://www.ncbi.nlm.nih.gov/clinvar/). To avoid duplicate counting of affected individuals, we reviewed all available information (including gender, country of origin, and phenotype) and contacted the authors when doubts persisted. Individuals reported more than once are indicated in Additional file 1: Table S1.

\section{Clinical evaluation}

Prospective clinical and psychological characterization was completed for 12 individuals seen at the Seaver Autism Center (S1-S4, S6-S8, S10-S14), including three previously reported (S1 and S3 [2] and S13 [26]). A battery of standardized assessments was used to examine ASD, intellectual functioning, adaptive behavior, language, motor skills, and sensory processing (see below). The medical evaluation included psychiatric, neurological, and clinical genetics examinations and medical record review. The evaluation of the individuals seen at Baylor College of Medicine (B1-B3) included parent interview, neurological examination, and medical record review. Their seizure phenotype and brain magnetic resonance imaging (MRI) findings were reported previously [24]. Two additional individuals (S5 and S9) received genetic testing through the Seaver Autism Center but were not evaluated clinically. Their caregivers completed surveys to capture developmental, medical, and behavioral health issues and were interviewed by phone.

\section{ASD phenotype}

Gold-standard ASD diagnostic testing included the Autism Diagnostic Observation Schedule, Second Edition (ADOS-2) [27], the Autism Diagnostic InterviewRevised (ADI-R) [28], and a clinical evaluation to assess Diagnostic and Statistical Manual for Mental Disorders, Fifth Edition (DSM-5) criteria for ASD [29]. The ADOS2 and ADI-R were administered and scored by research reliable raters, and the psychiatric evaluation was completed by a board-certified child and adolescent psychiatrist. The ADOS-2 is a semi-structured observational assessment that provides scores in the domains of social affect, restricted and repetitive behavior, and a total score. A comparison score ranging from 1 to 10 , with higher scores reflecting a greater number of symptoms, was calculated to examine symptom severity within each ADOS-2 domain and in total [30]. Nine individuals (S1-S4, S6, S8, S11, S13, S14) received module 1 of the ADOS, for children who are nonverbal or communicate using single words. Two individuals (S7, S10) received module 3 , for children who are verbally fluent. The ADI- $R$ is a structured caregiver interview that assesses ASD symptomatology within the domains of socialization, communication, and repetitive and restricted interests and behavior. A consensus diagnosis was determined for each participant based on results from the ADOS-2, ADI-R, and clinical evaluation using DSM-5.

\section{Intellectual functioning}

Global cognitive ability was measured using the Mullen Scales of Early Learning [31] $(n=10)$, the Stanford Binet Intelligence Scales, Fifth Edition [32] $(n=1)$, and the Differential Ability Scales, Second Edition (DAS-II) [33] $(n=1)$, depending on age and verbal ability. The Mullen is validated for children from birth to 68 months but is commonly used for older individuals with ID [34]. Developmental quotients were calculated using age equivalents divided by chronological age as has been done in previous studies [35]. For example, a nonverbal developmental quotient was computed by dividing the mean age equivalents on the visual reception and fine motor scales by the child's chronological age and then multiplying by 100 . The DAS-II is a measure of cognitive functioning that assesses a child's verbal reasoning, nonverbal reasoning, and spatial abilities. A general conceptual ability can be calculated to assess overall intellectual functioning. The Stanford-Binet Intelligence Scales, Fifth Edition is an intelligence test that produces a nonverbal intellectual quotient (IQ), verbal IQ, and full scale IQ based on performance across five scales: fluid reasoning, knowledge, quantitative reasoning, visualspatial, and working memory.

\section{Adaptive behavior}

The Vineland Adaptive Behavior Scales, Second Edition, Survey Interview Form (Vineland-II) [36] is a clinicianadministered interview that assesses adaptive behavior in the domains of communication, daily living, socialization, and motor skills. The Vineland-II was completed for 11 individuals. The motor domain is intended for children ages 6 years and under but was assessed in all individuals given significant motor delays in this population. The 
Vineland-II was also used in conjunction with cognitive testing to identify the presence and severity of ID.

\section{Language skills}

Language milestones were assessed during the ADI-R $(n=11)$ and the psychiatric evaluation. Current expressive and receptive language abilities were assessed using the Mullen $(n=10)$, Vineland-II $(n=11)$, MacArthur-Bates Communicative Development Inventories [37] $(n=10)$, Peabody Picture Vocabulary Test, Fourth Edition [38] $(n=3)$, and Expressive Vocabulary Test [39] $(n=2)$.

\section{Motor skills}

Motor milestones were assessed during the ADI-R $(n=11)$ and the psychiatric evaluation $(n=12)$. Current motor skills were assessed using the Vineland-II $(n=11)$ and Mullen $(n=10)$ fine and gross motor skills domains. The Beery Visual-Motor Integration Test, 6th Edition [40] was completed when appropriate $(n=2)$.

\section{Sensory processing}

Sensory processing was assessed using the Short Sensory Profile [41] and the Sensory Assessment for Neurodevelopmental Disorders (SAND) [42]. The Short Sensory Profile is a 38-item caregiver report form that investigates daily life sensory experiences. The SAND is a standardized assessment that includes a clinicianadministered observation and a 36-item corresponding caregiver interview. The scoring algorithm measures sensory hyperreactivity, hyporeactivity, and seeking behavior across visual, tactile, and auditory domains.

\section{Results}

\section{SHANK3 mutations}

We report 17 individuals (including two monozygotic twins) with SHANK3 mutations identified through WES or panel sequencing. The variants were distributed throughout the protein and included 13 frameshift, two nonsense, and one missense mutation (Table 1, Fig. 1a). Notably, we observed an identical frameshift mutation, c.3679dupG (p.Ala1227Glyfs*69), in three unrelated individuals. Mutations were confirmed to be de novo in 15 individuals and non-paternal or non-maternal in two (no DNA was available from the other two parents). In addition to a nonsense mutation, individual S13 carries a missense variant (p.Ser1291Leu) absent in the mother but present in the unaffected sister and in four individuals in the Genome Aggregation Database (gnomAD), suggesting it is likely benign, despite being predicted as damaging by several in silico tools (Additional file 1: Table S3). All other mutations are absent from the Exome Variant Server (EVS) and gnomAD. The missense mutation in S14 (p.Asp1672Tyr) affects a highly conserved residue and is predicted to be damaging by all algorithms used, including Polyphen-2, SIFT, PANTHER, MutPred2, Condel2, CADD, and M-CAP (Additional file 1: Table S3).

We also searched the literature and ClinVar for SHANK3 mutations and assessed their pathogenicity. Variants listed in Additional file 1: Table S1 meet the following criteria: (1) loss-of-function variants (frameshift, nonsense, and splice site), or de novo missense variants predicted to be deleterious by several bioinformatics predictors, and (2) absent from control databases (EVS and gnomAD). After removing cases ascertained or reported multiple times, we identified 60 additional individuals from 55 families with SHANK3 mutations classified as pathogenic or likely pathogenic according to ACMG [25]. All the mutations with parental samples available were de novo. Three families had multiple affected siblings, consistent with germline mosaicism [9, 22, 43]. Four de novo missense variants reported in children with ASD, ID, or infantile spasms (p.Thr337Ser, p.Ser1197Gly, p.Ala1214Pro, and p.Arg1255Gly) [15, 44-46] were classified as variants of uncertain significance because, although not present in controls, in silico predictions did not provide consistent evidence for pathogenicity (Additional file 1: Tables S1, S3). Given that SHANK3 is highly constrained against missense variation (Exome Aggregation Consortium Z score 4.92) [47], further studies are needed to determine the pathogenicity of these and other missense variants.

Three of the mutations in our cohort are recurrent, having been previously observed in unrelated individuals (Fig. 1a, Additional file 1: Table S1). The mutation in S6, p.Leu1142Valfs"153, was reported in a boy with ASD [13]. The mutation c.3679dupG (p.Ala1227Glyfs*69), shared by three of our patients (S7, S8, B1), is within a stretch of eight guanines and has been identified in three independent cases $[9,15,20]$. p.Arg1255Leufs*25, present in $\mathrm{S} 9$, has been reported in three unrelated patients $[13,21]$. The donor splice site at position c.2265 +1 is another hotspot: there are three individuals with a G>A substitution [16, 24, 48], and one with a deletion of the same G (c.2265+1delG), shown to result in a frameshift (p.Ser755Serfs*1) [11]. Overall, there were four recurrent and 56 private pathogenic/likely pathogenic mutations in SHANK3 (Fig. 1a, Additional file 1: Table S1).

We also searched for potentially deleterious variants inherited from unaffected parents or present in population controls (Additional file 1: Table S4). An inherited frameshift variant reported as pathogenic in two unrelated children with ASD [12, 49], and classified as damaging in the Human Gene Mutation Database, is in fact intronic when annotated in the correct reference sequence, NM_033517.1 [49], and is present 173 times 


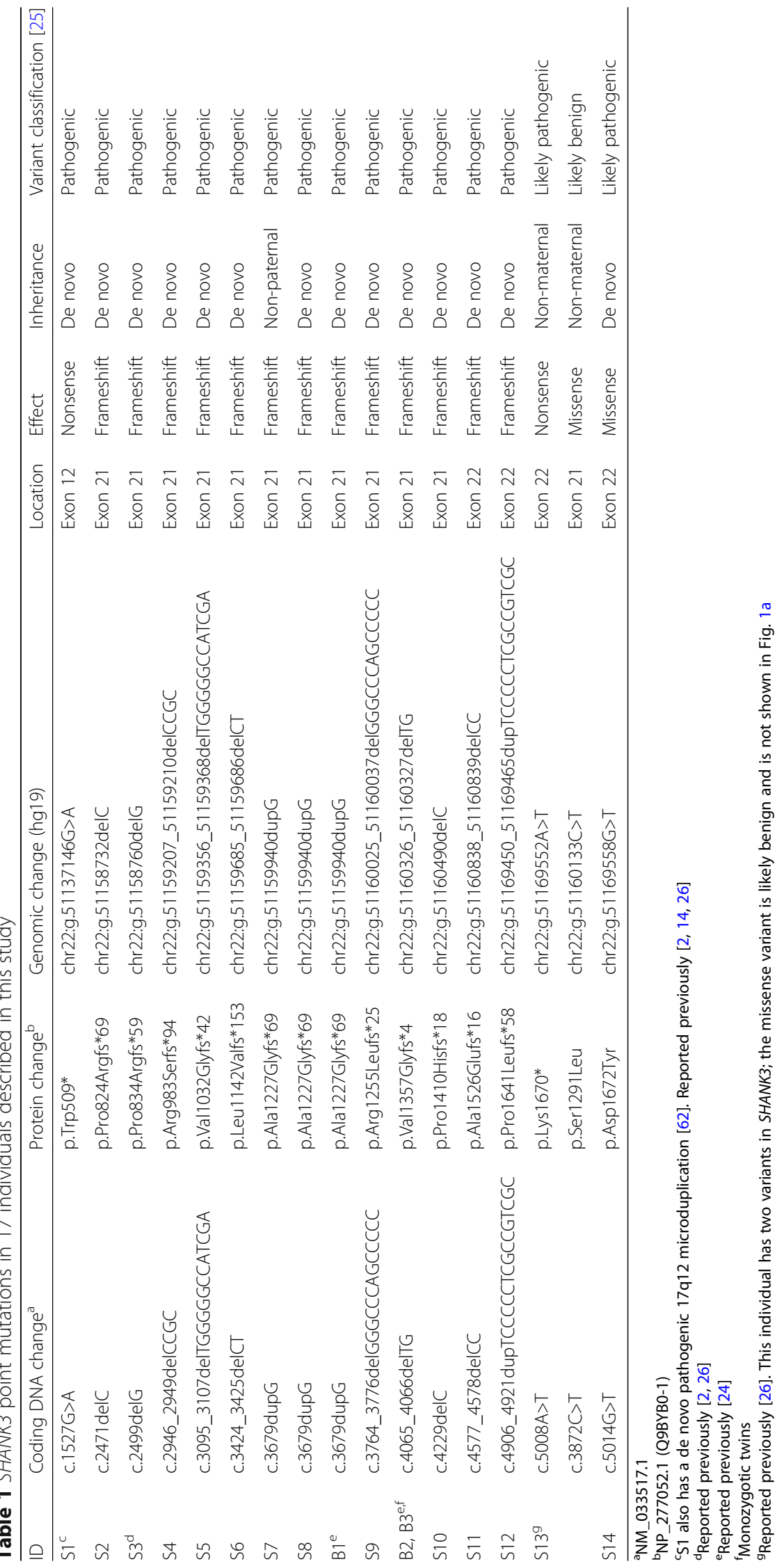




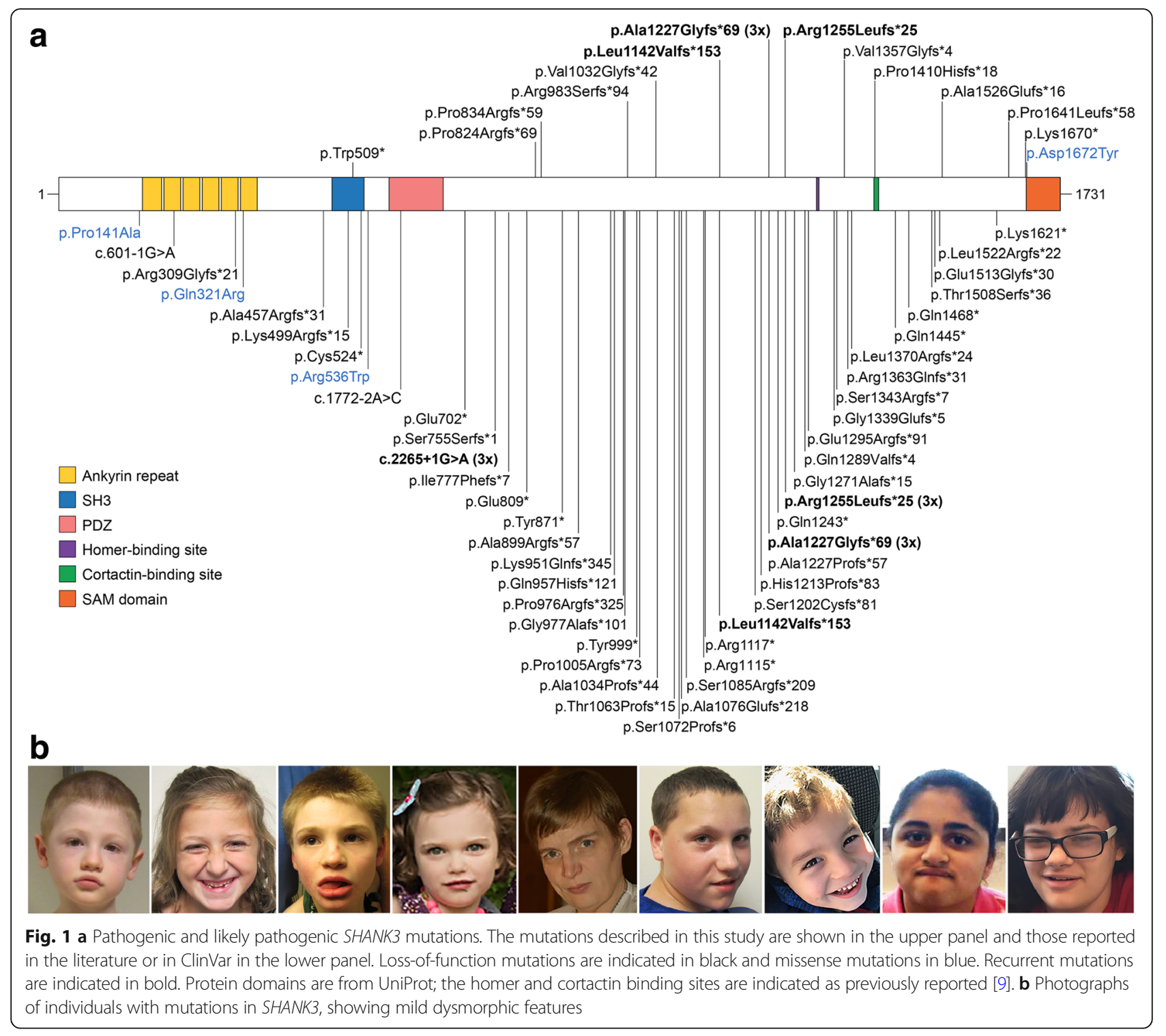

in gnomAD (chr22:g.51135705dupG, hg19). An inherited substitution in a splice region (c.1772-4G $>$ A) reported in ASD [12] is present seven times in gnomAD and is thus unlikely to be deleterious. gnomAD contains 21 variants predicted to be loss-of-function when annotated in the Ensembl canonical transcript ENST00000262795 (which is missing the beginning of exon 11 and contains three extra, unvalidated exons). When annotated in NM_033517.1, many of these variants are in fact intronic. The remaining 10 loss-of-function variants are all singletons; seven are flagged because they were found in sites covered in a limited number of individuals, which may indicate low-quality sites, one is located at the extreme 3 ' end, and one has an abnormal allele balance. These findings confirm that truncating variants in SHANK3 are highly penetrant and unlikely to be present in unaffected individuals.
Four in-frame deletions $[10,13,19,50]$ and one inframe insertion [50] in SHANK3 have been reported in ASD/ID (Additional file 1: Table S4). Three of these variants were inherited $[10,13,50]$, and one was found in two controls [50], suggesting that some short in-frame deletions or insertions may be tolerated. An in-frame deletion of five amino acids (p.Gly1453_Ala1457del) reported in an ASD proband and his unaffected mother [10] was detected in six individuals in the gnomAD database. gno$\mathrm{mAD}$ lists 15 in-frame deletions or insertions (after annotation in NM_033517.1); six are on multiallelic sites, and four others are flagged because of low coverage. Among the remaining in-frame variants, p.Glu1230del was observed in five individuals and p.Gly1518del in four (Additional file 1: Table S4). These findings indicate that at least some in-frame variants in SHANK3 can be present in seemingly unaffected individuals. 
Clinical phenotype of SHANK3 haploinsufficiency Phenotypic spectrum in the individuals from our cohort Detailed clinical information of the 17 individuals (9 males and 8 females, 3-42 years old at evaluation) is summarized in Tables 2 and 3 and Additional file 2: Table S5.

ASD Findings of ASD were widespread, with 69\% (11/ 16) receiving a diagnosis of ASD. Among the 11 individuals of the Seaver cohort who received ASD diagnostic testing and a psychiatric evaluation, $82 \%(9 / 11)$ met criteria for ASD on the ADOS and 73\% (8/11) met criteria for ASD on the ADI-R. A consensus diagnosis of ASD, accounting for both standardized assessments and clinical impression based on DSM-5 criteria, was reached in 73\% (8/11) (Additional file 2: Tables S5, S6). All three children who did not receive an ASD diagnosis (S3, S7, S10) showed relevant features, including two with scores above the ASD cutoff on the ADOS-2 or the ADI-R but not both. It is notable that two of these three individuals (S7 and S10) were verbally fluent with cognitive functioning on the cusp of mild ID/borderline cognitive functioning.

Additional behavioral findings All participants from the Seaver cohort had significant repetitive behaviors $(n=14)$, including hand flapping and stereotypic motor movements $(11 / 14,79 \%)$, chewing and teeth grinding $(7 / 14,50 \%)$, pica and mouthing of objects $(8 / 14,57 \%)$, and stereotypic vocalizations $(5 / 14,36 \%)$. The majority of participants were described as hyperactive $(11 / 17,65 \%)$, although the extent and severity of hyperactivity varied widely as did the extent of impulsivity and inattention. Participants were also prone to aggression $(8 / 17,47 \%)$ and self-injury $(3 / 15$, $20 \%)$, particularly when frustrated. Sleep disturbance was common $(10 / 17,59 \%)$.

Intellectual functioning ID was observed in all cases that received standardized testing $(n=13)$, with 10 cases falling in the range of a severe-to-profound ID and three cases in the mild range. Two individuals (B2, B3) who did not receive standardized testing were characterized as mildly intellectually disabled based on the extent of language and developmental delay. All individuals within the normed range of up to 68 months (S2, S4, S6, S14) achieved the lowest possible standard score on the Mullen Early Learning Composite $(<49,<1$ st percentile), indicating that the instrument reached its lower limit for reliable data collection ("floor" effect). Developmental quotients (DQ) were calculated for all individuals (excluding the 42-year-old individual) and ranged from 6.7 to 30 (mean $\pm \mathrm{SD}, 15.6 \pm 8.0$ ). Verbal $\mathrm{DQ}$ ranged from 9.2 to $35(19.9 \pm 9.2)$, and nonverbal DQ ranged from 3.1 to $25.8(11.39 \pm 7.16)$ (Additional file 2: Table S6). Results from three additional cases who received other cognitive measures (S7, S10, and B1) indicated the presence of mild-to-moderate ID (Table 2).

Adaptive behavior Results from the Vineland-II indicated that adaptive functioning was consistent with cognitive functioning (Additional file 2: Table S5). Overall, motor skills and socialization skills were better developed than communication and daily living skills. Two children (S7, S10) fell within the borderline range; all others fell below the first percentile.

Language skills Language impairment was prominent (17/17, 100\%); results are summarized in Table 2. All subjects were delayed in achieving language milestones. With regard to current language abilities, the ADOS-2 $(n=11)$ indicated that five individuals used no words, three used $<5$ recognizable words or word approximations, one used mostly single words, and two used complex speech with frequent grammatical errors. Receptive and expressive language were equally delayed (Additional file 2: Table S7). Three individuals (S7, S10, S14) were administered the Peabody Picture Vocabulary Test and achieved scores between $<1$ st and 7 th percentiles. Two of these individuals (S7, S10) achieved scores of 70 (2nd percentile) on the Expressive Vocabulary Test, indicating that despite fluent speech, expressive language abilities were significantly delayed relative to same-aged peers. Two of the Baylor participants were also reported to speak in sentences, but one was mostly echolalic.

Motor skills Most individuals achieved motor milestones on time, despite significant fine and gross motor delays in all participants at the time of evaluation. Hypotonia $(16 / 17,94 \%)$ and gait abnormalities $(14 / 17,82 \%)$ were present in the majority of individuals. Gross motor skills were significantly better developed than fine motor skills ( $n=9, p=0.02$ for both the Mullen and the Vineland-II, Wilcoxon signed-rank test; Additional file 2: Table S7). Two individuals (S7, S10) were administered the Beery Visual-Motor Integration Test and received standard scores of 45 and 65 , respectively, which is indicative of visual-motor deficits.

Sensory processing According to parent report, 16 of 17 participants had increased pain tolerance (94\%). Results from the Sensory Assessment for Neurodevelopmental Disorders $(n=10)$ and clinical observation indicated that sensory hyporeactivity (i.e., underresponsiveness to stimuli) was prominent. These findings are consistent with the results from the Short Sensory Profile $(n=11)$, indicating high scores in the 


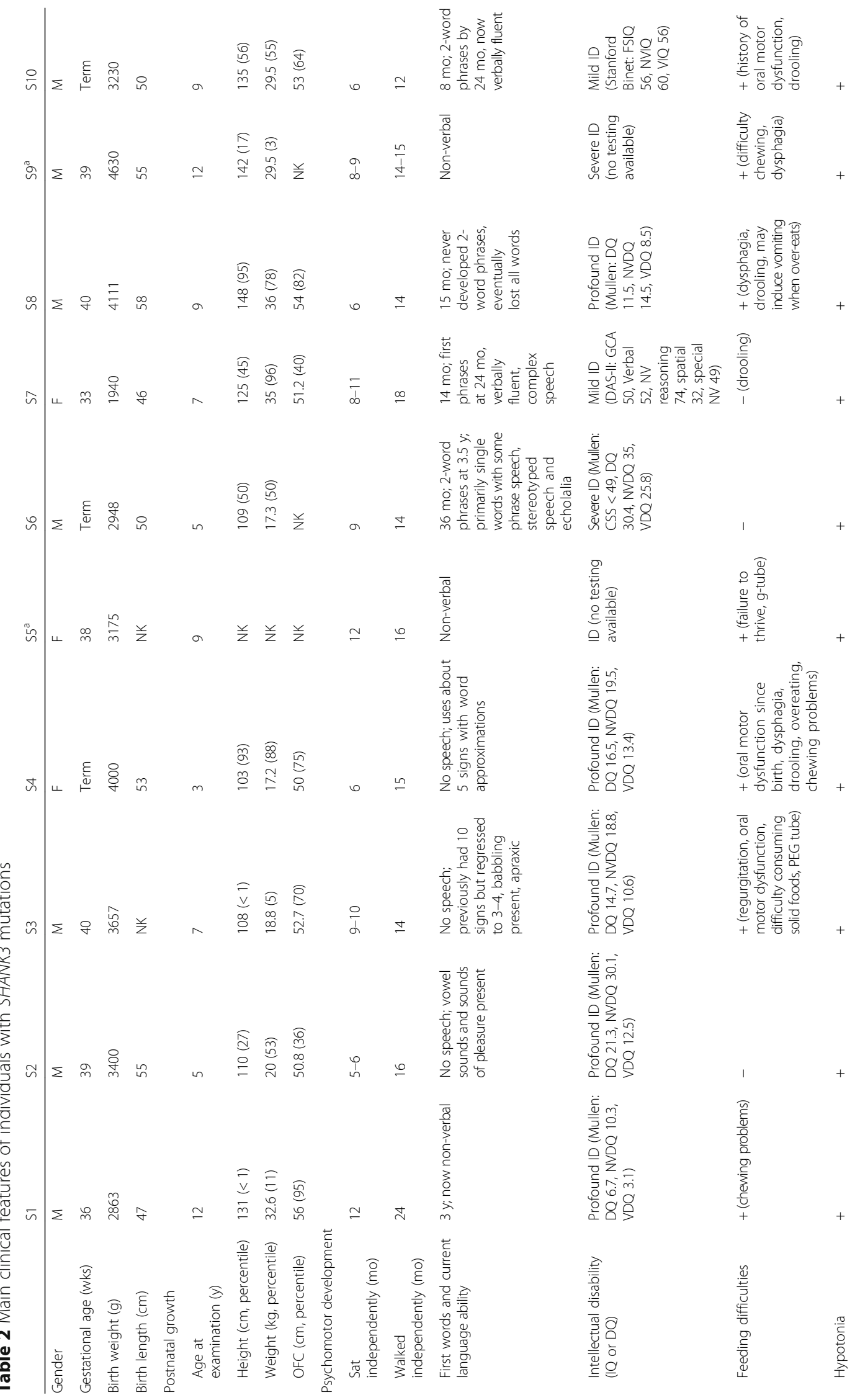




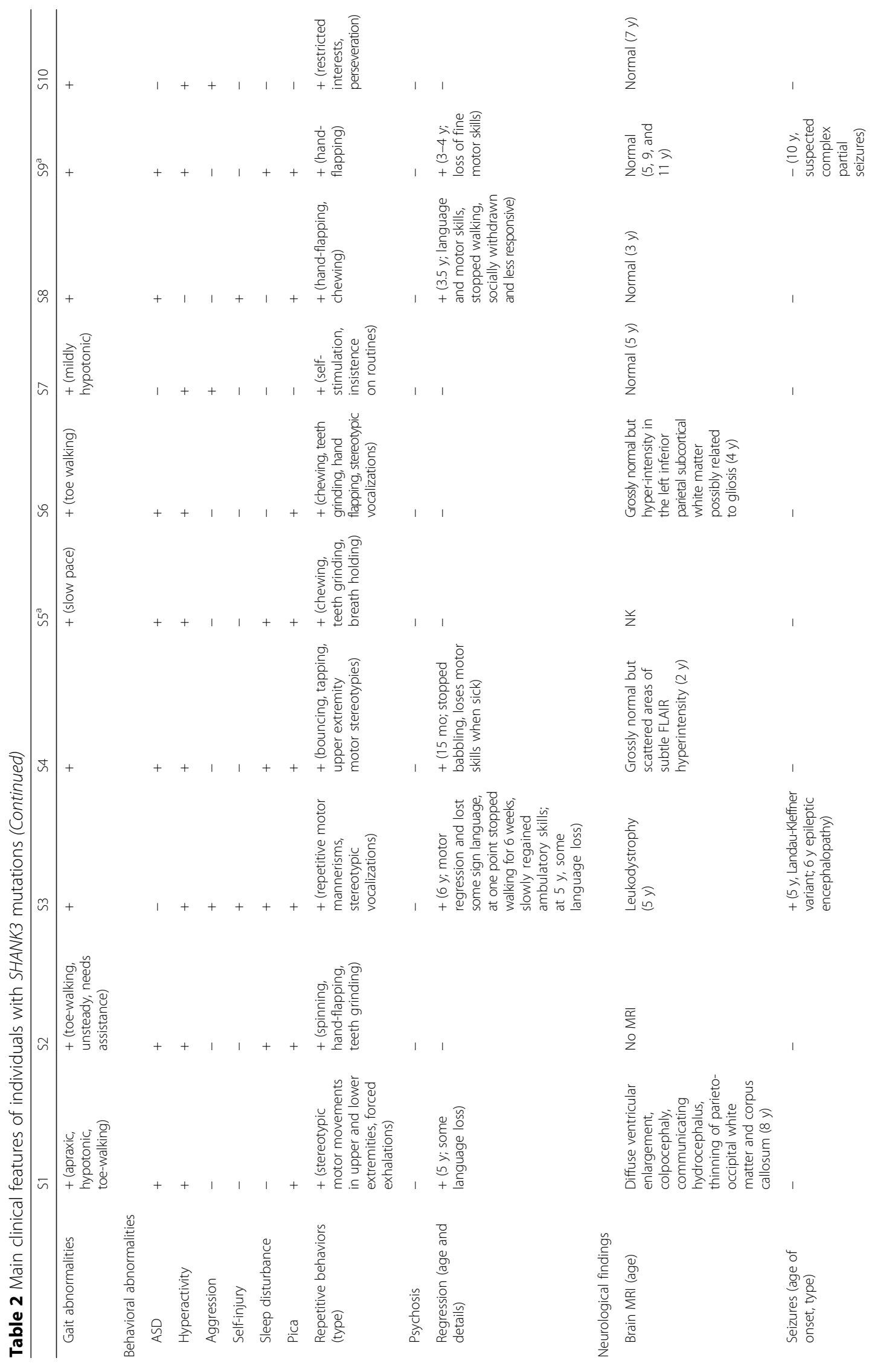


De Rubeis et al. Molecular Autism (2018) 9:31

Page 10 of 20

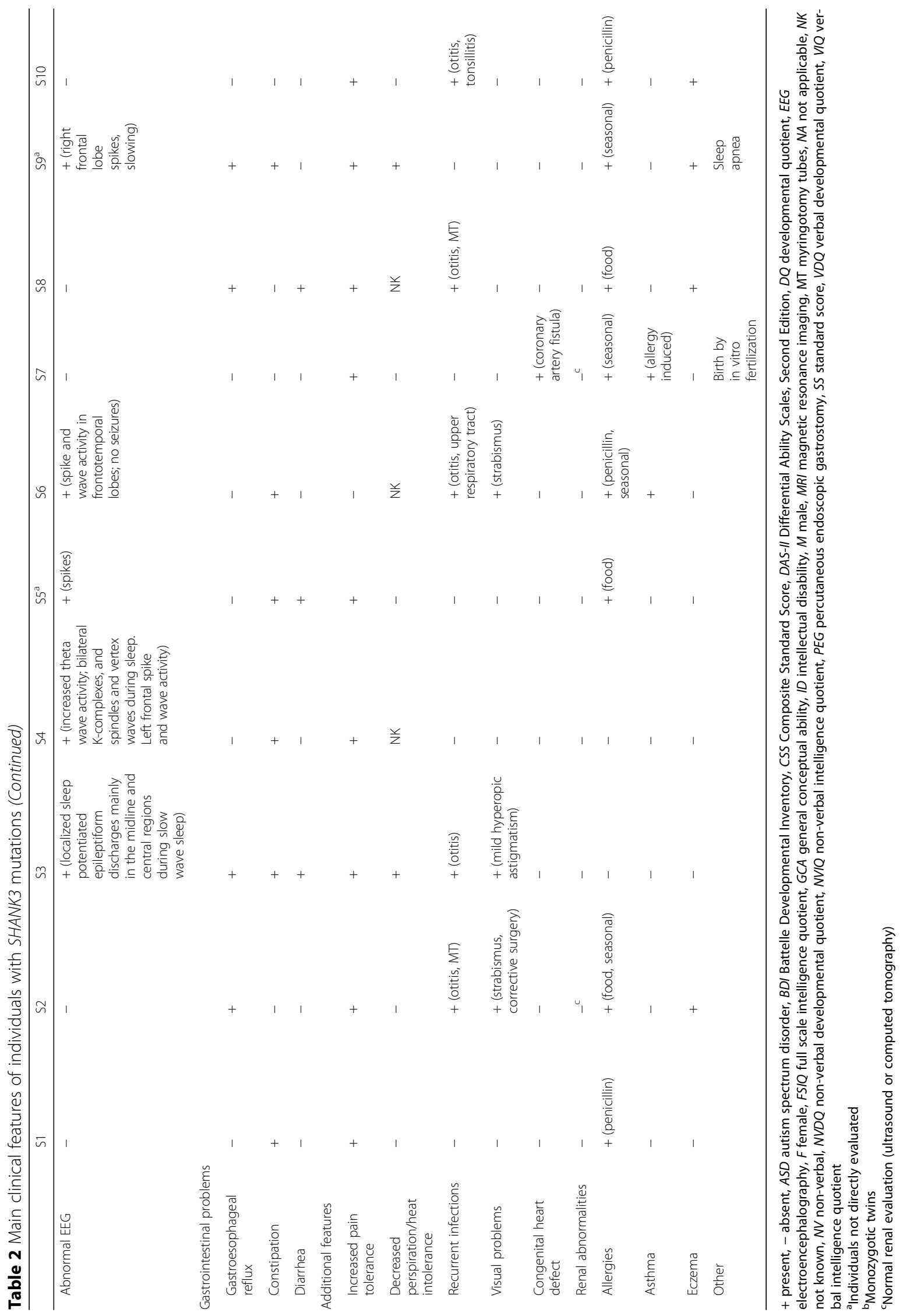




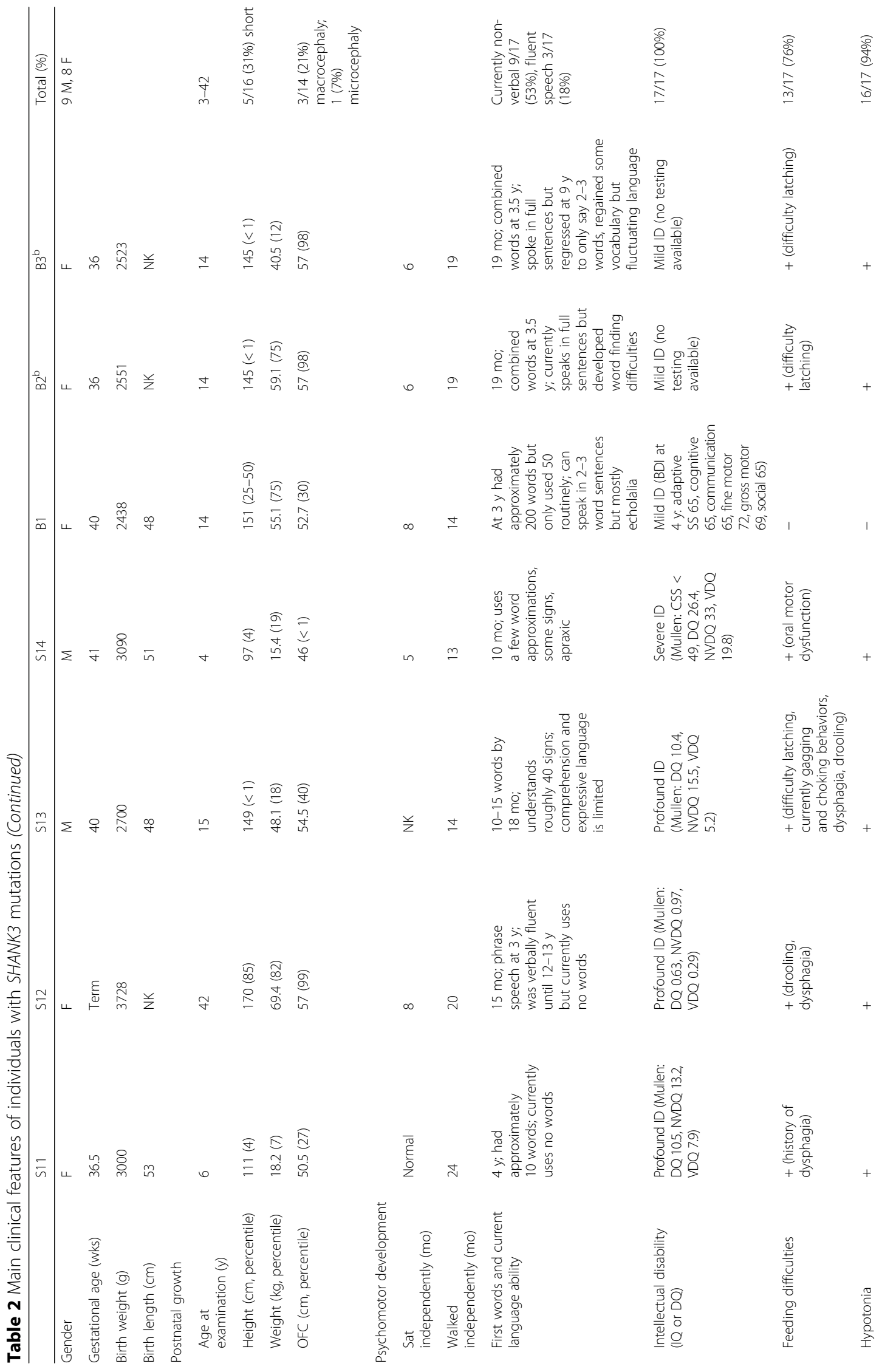


De Rubeis et al. Molecular Autism (2018) 9:31

Page 12 of 20

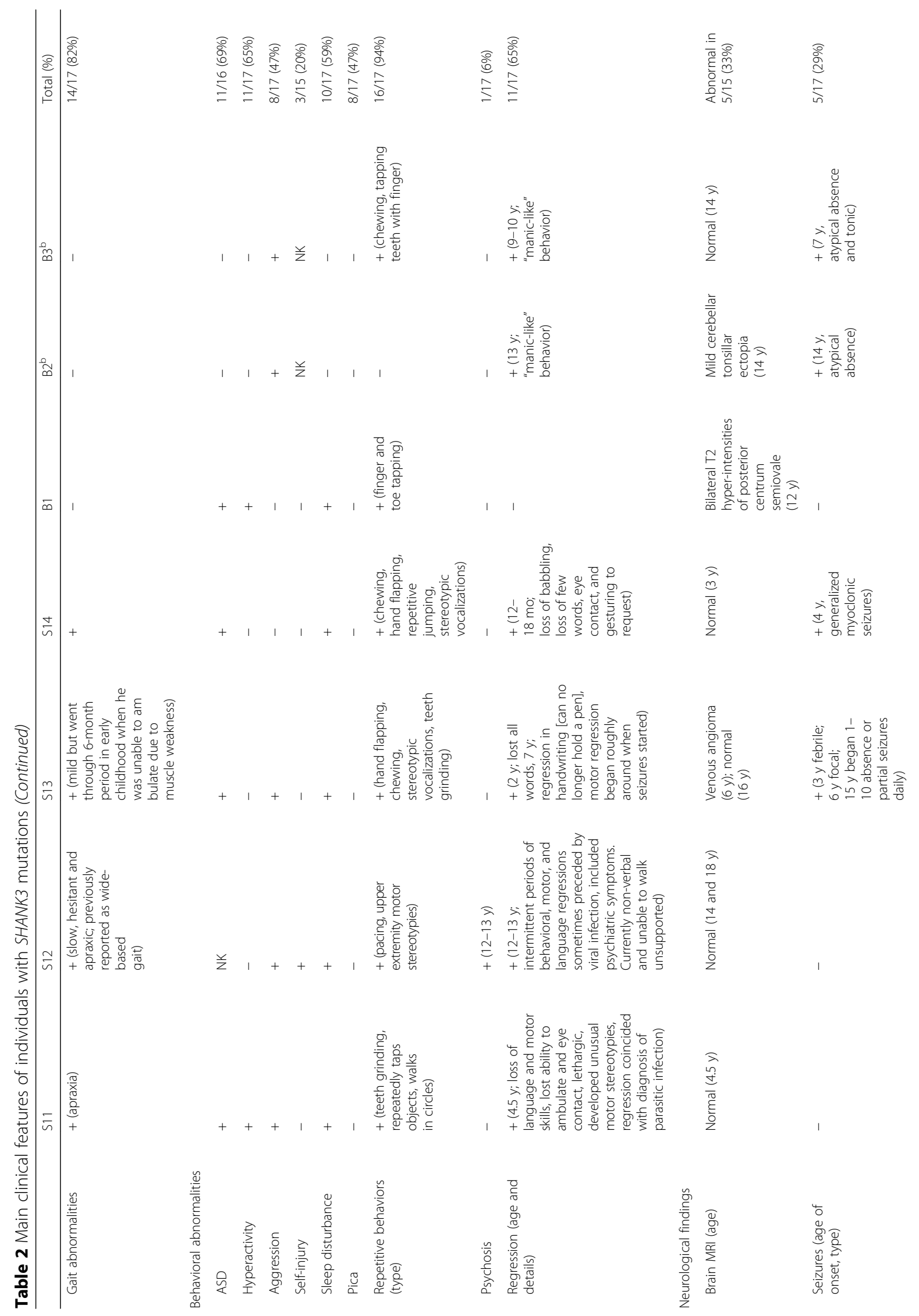




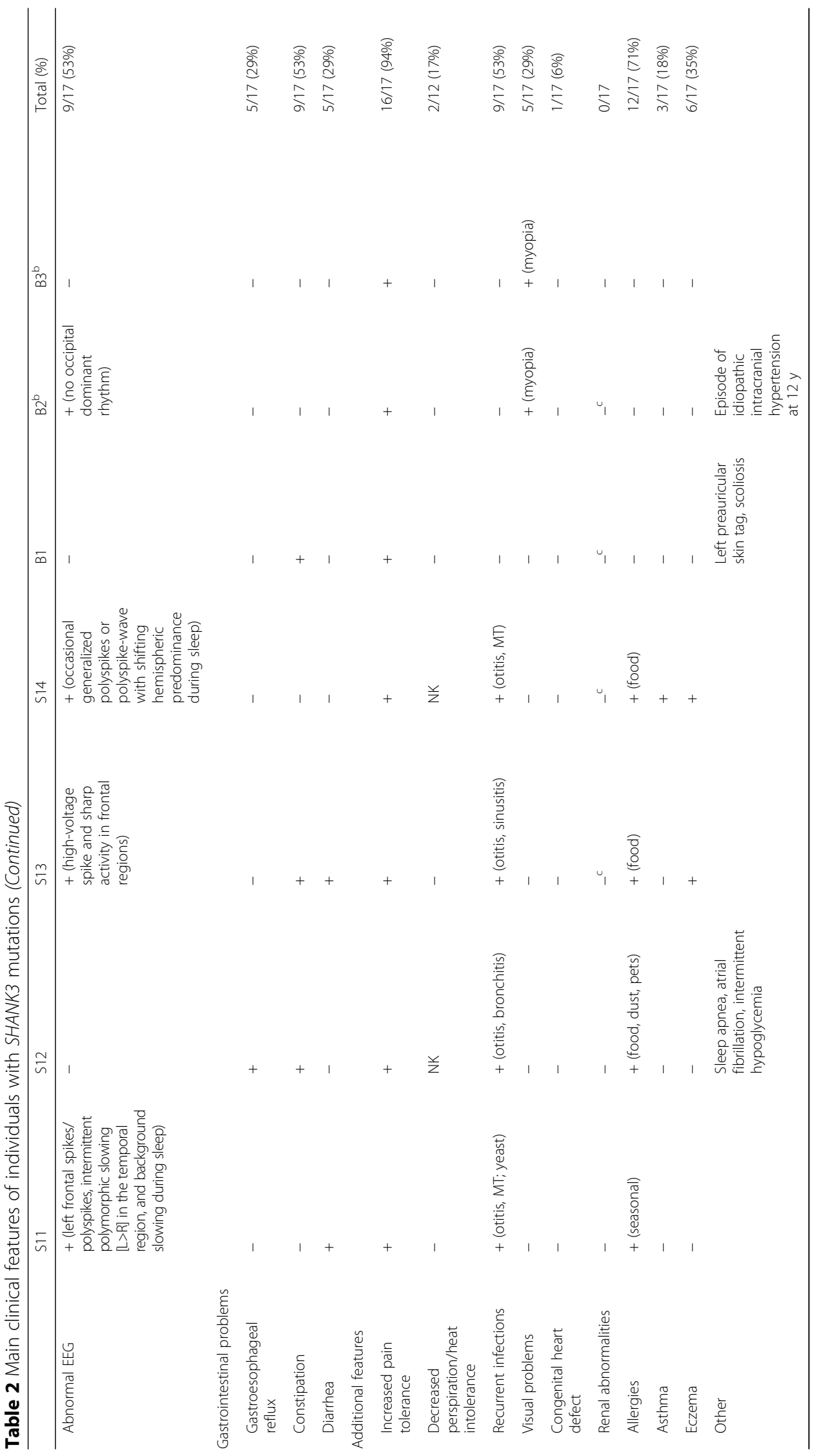


Table 3 Dysmorphic features in individuals with SHANK3 mutations

\begin{tabular}{|c|c|c|c|c|c|c|c|c|c|c|c|c|}
\hline & $\mathrm{S} 1^{\mathrm{a}}$ & S2 & S3 & S4 & S7 & S8 & $\mathrm{S} 10$ & S11 & $\mathrm{S} 12$ & $\mathrm{~S} 13$ & S14 & Total (\%) \\
\hline Gender & $M$ & $M$ & M & $\mathrm{F}$ & $\mathrm{F}$ & M & M & $\mathrm{F}$ & $\mathrm{F}$ & M & M & $7 \mathrm{M}, 4 \mathrm{~F}$ \\
\hline Age at examination (years) & 12 & 5 & 7 & 3 & 7 & 9 & 9 & 6 & 42 & 15 & 4 & $3-42$ \\
\hline \multicolumn{13}{|l|}{ Craniofacial features } \\
\hline Microcephaly ${ }^{\mathrm{b}}$ & - & - & - & - & - & - & - & - & - & - & + & $1 / 11(9 \%)$ \\
\hline Macrocephaly ${ }^{c}$ & - & - & - & - & - & - & - & - & + & - & - & $1 / 11(9 \%)$ \\
\hline Dolichocephaly & - & - & + & - & - & - & - & - & - & - & - & $1 / 11(9 \%)$ \\
\hline Synophrys & - & - & - & - & - & - & - & - & - & - & - & 0 \\
\hline Sparse eyebrows & - & - & - & + & - & - & - & - & - & + & - & $2 / 11(18 \%)$ \\
\hline Long eyelashes & + & + & - & + & + & + & - & + & + & + & - & 8/11 (73\%) \\
\hline Periorbital fullness & + & + & - & + & - & - & - & - & - & + & - & $4 / 11(36 \%)$ \\
\hline Deep set eyes & - & + & - & + & - & + & + & + & - & - & - & $5 / 11(45 \%)$ \\
\hline Ptosis & - & - & - & - & - & - & - & - & - & - & - & 0 \\
\hline Epicanthal folds & + & + & - & + & - & - & + & - & - & - & + & $5 / 11(45 \%)$ \\
\hline Hypertelorism & - & - & - & - & - & - & - & - & - & - & - & 0 \\
\hline Wide nasal bridge & + & + & - & + & + & + & - & - & - & - & + & $6 / 11(55 \%)$ \\
\hline Bulbous nose & - & + & - & + & + & + & - & - & - & + & + & $6 / 11(55 \%)$ \\
\hline Anteverted nares & - & - & - & - & + & - & - & - & - & - & - & 1/11 (9\%) \\
\hline Full cheeks & - & - & - & + & - & - & - & - & - & + & - & $2 / 11(18 \%)$ \\
\hline Malar hypoplasia & + & + & - & + & - & - & - & - & + & + & - & $5 / 11(45 \%)$ \\
\hline Thin upper vermillion & - & + & + & - & - & - & - & + & - & - & - & $3 / 11(27 \%)$ \\
\hline Thick lower vermillion & + & + & + & - & - & - & - & - & - & - & - & $3 / 11(27 \%)$ \\
\hline Short philtrum & - & - & - & - & - & - & - & - & - & - & - & 0 \\
\hline Long philtrum & - & + & - & - & - & - & + & - & + & - & - & $3 / 11(27 \%)$ \\
\hline Malocclusion & + & + & - & + & + & + & + & - & + & - & - & $7 / 11(64 \%)$ \\
\hline High arched palate & + & + & - & + & - & - & + & - & $\mathrm{NE}$ & + & + & $6 / 10(60 \%)$ \\
\hline Ear anomalies & - & - & - & $\begin{array}{l}+ \text { (low set } \\
\text { ears) }\end{array}$ & - & - & $\begin{array}{l}+ \text { (overfolded } \\
\text { helix) }\end{array}$ & - & $\begin{array}{l}+(\text { fleshy } \\
\text { ears) }\end{array}$ & - & $\begin{array}{l}+ \text { (prominent } \\
\text { ears) }\end{array}$ & $4 / 11(36 \%)$ \\
\hline Micrognathia & - & - & - & - & - & - & - & - & - & - & - & 0 \\
\hline Macrognathia & + & - & - & - & + & - & - & - & + & - & - & $3 / 11(27 \%)$ \\
\hline Pointed chin & + & - & - & + & + & - & + & + & + & + & - & $7 / 11(64 \%)$ \\
\hline \multicolumn{13}{|l|}{ Hand and feet anomalies } \\
\hline Large fleshy hands & - & - & - & + & - & - & - & - & - & + & + & $3 / 11(27 \%)$ \\
\hline 5th finger clinodactyly & + & + & - & + & + & + & + & - & + & + & + & 9/11 (82\%) \\
\hline Partial syndactyly of toes $2-3$ & - & + & - & + & - & - & + & - & - & + & + & $5 / 11(45 \%)$ \\
\hline Sandal gap & + & + & + & NE & + & + & - & $\mathrm{NE}$ & - & + & - & $6 / 9(67 \%)$ \\
\hline $\begin{array}{l}\text { Hypoplasia of distal phalanges } \\
\text { of } 5 \text { th finger }\end{array}$ & - & - & - & - & - & - & - & - & + & + & - & $2 / 11(18 \%)$ \\
\hline \multicolumn{13}{|l|}{ Ectodermal anomalies } \\
\hline Hypertrichosis & - & - & - & - & - & - & - & - & - & - & - & 0 \\
\hline Abnormal hair whorl & - & - & - & - & - & - & - & + & - & + & - & $2 / 11(18 \%)$ \\
\hline $\begin{array}{l}\text { Hypoplastic/dysplastic } \\
\text { toenails }\end{array}$ & + & - & - & + & - & + & - & + & + & - & + & $6 / 11(55 \%)$ \\
\hline $\begin{array}{l}\text { Hypoplastic/dysplastic } \\
\text { fingernails }\end{array}$ & + & + & - & - & - & - & - & - & - & - & - & $2 / 11(18 \%)$ \\
\hline
\end{tabular}


Table 3 Dysmorphic features in individuals with SHANK3 mutations (Continued)

\begin{tabular}{|c|c|c|c|c|c|c|c|c|c|c|c|c|}
\hline & $S 1^{\mathrm{a}}$ & S2 & S3 & S4 & S7 & S8 & $\mathrm{S} 10$ & $\mathrm{~S} 11$ & $\mathrm{~S} 12$ & $\mathrm{~S} 13$ & S14 & Total (\%) \\
\hline \multicolumn{13}{|l|}{ Other features } \\
\hline $\begin{array}{l}\text { Short stature/delayed } \\
\text { growth }^{d}\end{array}$ & + & - & + & - & - & - & - & - & - & + & - & $3 / 11(27 \%)$ \\
\hline $\begin{array}{l}\text { Tall stature/accelerated } \\
\text { growth }^{\mathrm{e}}\end{array}$ & - & - & - & - & - & - & - & - & - & - & - & 0 \\
\hline Hyperextensibility & - & - & - & + & + & + & + & - & NE & + & + & $6 / 10(60 \%)$ \\
\hline Sacral dimple & - & - & $\mathrm{NE}$ & - & - & - & - & - & - & - & - & 0 \\
\hline Scoliosis & - & - & - & - & - & - & - & - & - & + & - & $1 / 11(9 \%)$ \\
\hline Total dysmorphic features & 15 & 16 & 5 & 18 & 10 & 9 & 10 & 6 & 11 & 17 & 11 & $11 / 11(100 \%)$ \\
\hline
\end{tabular}

Only individuals that underwent a detailed evaluation by a clinical geneticist are shown

+ present, - absent, $F$ female, $M$ male, $N E$ not evaluated

${ }^{a} \mathrm{~S} 1$ also has a de novo pathogenic $17 \mathrm{q} 12$ microduplication [62]

${ }^{b}$ Head circumference $<3$ rd percentile

'Head circumference $>$ 98th percentile

${ }^{\mathrm{d} H e i g h t}<3$ rd percentile

${ }^{\mathrm{e}}$ Height $>98$ th percentile

underresponsive/seeks sensation domain (10/11) and the low energy/weak domain (9/11).

Neurological findings Seizures were reported in five individuals $(5 / 17,29 \%)$, including febrile $(n=1)$, absence $(n$ $=3$ ), focal $(n=1)$, and generalized seizures $(n=2)$ (one individual had febrile, absence, and focal seizures) (Table 2). Age of onset ranged from 4 to 14 years $(7.2 \pm 4)$. Nine individuals had an abnormal electroencephalography (EEG) (9/17, 53\%), including five without clinical seizures. MRI in 15 individuals revealed abnormal findings in five (33\%), including white matter abnormalities $(n=3)$, venous angioma $(n=1)$, and mild cerebellar ectopia $(n=1)$.

Regression For the purpose of this manuscript, we only document regression in patients who clearly and consistently acquired skills for a prolonged period of time and then lost these skills, either permanently or for an extended period. Regression, occurring at various stages of development from early childhood to early adolescence, and affecting language, motor, and behavioral domains, was reported in 11 of 17 cases (65\%). At least two caregivers noted regression that was triggered by infection and one reported seizures preceding the onset of regression.

Other medical conditions Gastrointestinal problems were common, including gastroesophageal reflux (5/17, $29 \%)$, constipation $(9 / 17,53 \%)$, and diarrhea $(5 / 17,29 \%)$. Feeding problems were also common (13/17, 76\%), including dysphagia and chewing difficulties; two individuals required placement of a gastrostomy tube. Recurrent infections were reported in 53\% (9/17) of individuals, most often affecting the ears. Visual problems, and strabismus in particular, have been described in carriers of 22q13.3 deletions [2, 4, 51] and were present in $29 \%(5 / 17)$ of patients, including strabismus $(n=2)$, myopia $(n=2)$, and astigmatism $(n=1)$. Renal or urinary tract abnormalities, reported in $26-40 \%$ of cases with $22 q 13$ deletions [2, 4], were absent in our cohort. Similarly, congenital heart defects, reported in $3-13 \%$ of patients with 22q13 deletions [2, 52], were uncommon; one individual had a coronary artery fistula that did not require surgical intervention. Lymphedema, cellulitis, precocious or delayed puberty, hearing problems, and hypothyroidism have been reported in cases with $22 \mathrm{q} 13$ deletions $[2,4]$ but were not present in individuals with SHANK3 mutations (Table 4).

Dysmorphic features Dysmorphology examinations were performed on 11 individuals from the Seaver cohort, using a PMS-specific checklist (Table 3, Fig. 1b). All had at least five usually mild dysmorphic features (range 5-18), without a distinctive facial gestalt. In general, findings were consistent with those reported in patients with 22q13 deletions [2, 4-6]. However, some features were more common than previously reported, including fifth finger clinodactyly (9/11, 82\%), malocclusion (7/11, 64\%), and wide nasal bridge $(6 / 11,55 \%)$ (Table 4$)$. The use of a PMS-specific checklist could account in part for the higher frequency with which certain features were noted. Other features present in over $50 \%$ of the individuals were long eyelashes, bulbous nose, high arched palate, pointed chin, hyperextensibility, dysplastic toenails, and sandal gap.

\section{Phenotype of individuals with SHANK3 mutations in the literature}

The clinical features of 45 previously published individuals with pathogenic or likely pathogenic SHANK3 
Table 4 Clinical features in individuals with SHANK3 mutations as compared to 22q13 deletions including SHANK3

\begin{tabular}{|c|c|c|}
\hline Clinical features & $\begin{array}{l}\text { Individuals with SHANK3 } \\
\text { mutations (current study) }\end{array}$ & $\begin{array}{l}\text { Individuals with } \\
22 \text { 13 deletions [2] }\end{array}$ \\
\hline Intellectual disability & 17/17 (100\%) & 29/30 (97\%) \\
\hline ASD & $11 / 16(69 \%)$ & 26/30 (87\%) \\
\hline Verbally fluent & $3 / 17(18 \%)$ & $0 / 30$ \\
\hline Repetitive behaviors & 16/17 (94\%) & $30 / 30(100 \%)$ \\
\hline Hyperactivity & $11 / 17(65 \%)$ & $14 / 30(47 \%)$ \\
\hline Aggression & $8 / 17(47 \%)$ & 13/30 (43\%) \\
\hline Sleep disturbance & 10/17 (59\%) & $12 / 30(40 \%)$ \\
\hline Hypotonia & 16/17 (94\%) & 23/30 (77\%) \\
\hline Gait abnormalities & 14/17 (82\%) & 13/14 (93\%) \\
\hline Seizures & $5 / 17(29 \%)$ & $12 / 30(40 \%)$ \\
\hline Abnormal brain MRI & 5/15 (33\%) & 18/26 (69\%) \\
\hline Short stature ${ }^{a}$ & $5 / 16(31 \%)$ & $3 / 30(10 \%)$ \\
\hline Tall stature ${ }^{b}$ & $0 / 16$ & $1 / 30(3 \%)$ \\
\hline Microcephaly ${ }^{c}$ & $1 / 14(7 \%)$ & $2 / 30(7 \%)$ \\
\hline Macrocephaly ${ }^{d}$ & $3 / 14(21 \%)$ & $9 / 30(30 \%)$ \\
\hline Dolichocephaly & 1/11 (9\%) & $7 / 30(23 \%)$ \\
\hline $\begin{array}{l}\text { Sparse hair/abnormal } \\
\text { whorl }\end{array}$ & $2 / 11(18 \%)$ & $5 / 30(17 \%)$ \\
\hline Long eyelashes & 8/11 (73\%) & 13/30 (43\%) \\
\hline Periorbital fullness & 4/11 (36\%) & $8 / 30(27 \%)$ \\
\hline Hypertelorism & $0 / 11$ & $3 / 30(10 \%)$ \\
\hline Deep set eyes & $5 / 11(45 \%)$ & $2 / 30(7 \%)$ \\
\hline Ptosis & $0 / 11$ & $2 / 30(7 \%)$ \\
\hline Epicanthal folds & $5 / 11(45 \%)$ & $9 / 30(30 \%)$ \\
\hline Strabismus & $2 / 17(12 \%)$ & $3 / 30(10 \%)$ \\
\hline Wide nasal bridge & $6 / 11(55 \%)$ & $4 / 30(13 \%)$ \\
\hline Bulbous nose & $6 / 11(55 \%)$ & $15 / 30(50 \%)$ \\
\hline Full cheeks & $2 / 11(18 \%)$ & $8 / 30(27 \%)$ \\
\hline Malar hypoplasia & $5 / 11(45 \%)$ & $3 / 30(10 \%)$ \\
\hline Long philtrum & $3 / 11(27 \%)$ & $5 / 30(17 \%)$ \\
\hline Malocclusion & $7 / 11(64 \%)$ & $5 / 30(17 \%)$ \\
\hline Widely spaced teeth & $0 / 11$ & $1 / 30(3 \%)$ \\
\hline High arched palate & $6 / 10(60 \%)$ & $8 / 30(27 \%)$ \\
\hline Ear anomalies & 4/11 (36\%) & $13 / 30(43 \%)$ \\
\hline Pointed chin & $7 / 11(64 \%)$ & $7 / 30(23 \%)$ \\
\hline Large fleshy hands & $3 / 11(27 \%)$ & $17 / 30(57 \%)$ \\
\hline 5th finger clinodactyly & $9 / 11(82 \%)$ & $3 / 30(10 \%)$ \\
\hline Syndactyly of toes 2-3 & $5 / 11(45 \%)$ & $3 / 30(10 \%)$ \\
\hline $\begin{array}{l}\text { Hypoplastic/dysplastic } \\
\text { nails }\end{array}$ & $7 / 11(64 \%)$ & $11 / 30(37 \%)$ \\
\hline Hyperextensibility & $6 / 10(60 \%)$ & $8 / 30(27 \%)$ \\
\hline Scoliosis & 1/11 (9\%) & $7 / 30(23 \%)$ \\
\hline Sacral dimple & $0 / 10$ & $4 / 30(13 \%)$ \\
\hline
\end{tabular}

Table 4 Clinical features in individuals with SHANK3 mutations as compared to 22q13 deletions including SHANK3 (Continued)

\begin{tabular}{lll}
\hline Clinical features & $\begin{array}{l}\text { Individuals with SHANK3 } \\
\text { mutations (current study) }\end{array}$ & $\begin{array}{l}\text { Individuals with } \\
\text { 22q13 deletions [2] }\end{array}$ \\
\hline Gastroesophageal reflux & $5 / 17(29 \%)$ & $13 / 30(43 \%)$ \\
Constipation/diarrhea & $11 / 17(65 \%)$ & $11 / 30(37 \%)$ \\
Increased pain tolerance & $16 / 17(94 \%)$ & $26 / 30(87 \%)$ \\
Recurrent infections & $9 / 17(53 \%)$ & $16 / 30(53 \%)$ \\
Renal abnormalities & $0 / 17$ & $12 / 30(40 \%)$ \\
Congenital heart defect & $1 / 17(6 \%)$ & $1 / 30(3 \%)$ \\
Hypothyroidism & $0 / 17$ & $1 / 30(3 \%)$ \\
Lymphedema & $0 / 17$ & $7 / 30(23 \%)$ \\
\hline
\end{tabular}

ASD autism spectrum disorder

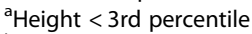

${ }^{\text {bHeight }}>98$ th percentile

'Head circumference $<3$ rd percentile

${ }^{\mathrm{d}}$ Head circumference $>98$ th percentile

variants are summarized in Additional file 1: Table S2. (Fifteen individuals reported only in ClinVar are included in Additional file 1: Table S1 where we summarize the allelic spectrum but are not included here because no phenotype information was available for them.) Although only limited information was available for most cases, the phenotype was consistent with that observed in our cohort, including ID (33/33, 100\%), severe language impairment (22/23, 96\%), ASD (26/34, $76 \%)$, hypotonia $(8 / 12,67 \%)$, seizures $(17 / 30,57 \%)$, and dysmorphic features (13/21, 62\%). Regression was reported in 11 individuals.

\section{Discussion}

This is the first study to comprehensively describe the phenotype in patients with PMS due to SHANK3 point mutations. Our findings demonstrate that loss of SHANK3 alone is sufficient to produce the characteristic features of PMS, including ID, ASD, severe speech impairment, hypotonia, epilepsy, motor skills deficits, feeding difficulties, mild dysmorphic features, increased pain tolerance, gastrointestinal problems, and neuroimaging abnormalities. In addition, we advance the understanding of the genetic architecture of PMS and, in so doing, provide information to aid in the interpretation of SHANK3 variants.

\section{Genetic findings}

Findings in our cohort and in previously reported patients indicate that SHANK3 mutations are fully penetrant. The identification of three families with SHANK3 mutations in multiple siblings due to germline mosaicism $(5 \%, 3 / 57)$ $[9,22,43]$ has important implications for genetic counseling. Of note, we identified four recurrent mutations in SHANK3, including p.Leu1142Valfs*153, p.Ala1227Glyfs*69, p.Arg1255Leufs"25, and c.2265+1G>A. The 
most common mutation, c.3679dupG (p.Ala1227Glyfs*69), identified in six individuals, is due to the duplication of a guanine in a stretch of eight guanines, indicating that this segment is prone to replication errors. Functional studies on several of the truncating mutations described here (p.Trp509* in S1, p.Pro834Argfs*59 in S3, p.Lys1670* in S13, and p.A1227Gf**69 in S7, S8, and B1) provide further support for their deleterious effects $[9,26,53,54]$.

Although the majority of pathogenic/likely pathogenic SHANK3 variants identified to date are truncating, the interpretation of missense variants remains difficult. Missense variant assessment relies on inheritance, segregation within families, frequency in population databases, functional studies, and computational predictions of pathogenicity (see ACMG guidelines [25]). In the case of SHANK3, in silico prediction programs often provide contradictory results (Additional file 1: Table S3). Functional studies could help determine the pathogenicity of missense substitutions; however, previous in vitro analyses have identified synaptic defects associated with missense variants in ASD inherited from healthy parents and found in control databases $[9,53,54]$; hence, more discriminatory functional approaches will need to be developed.

\section{ASD, ID, language, and motor skills}

Our results demonstrate the high prevalence of ASD in individuals with PMS resulting from SHANK3 mutations, similar to our previous findings in individuals with 22q13 deletions [2]. The ADOS and ADI-R provided important information regarding ASD features, even in individuals with low mental ages; however, clinical evaluation and consensus discussion proved necessary to determine which individuals did not meet criteria for ASD. Negative ASD findings in the two verbally fluent individuals raise questions about the relationship between ASD diagnosis and severe global developmental delay. Interestingly, in spite of severe-to-profound ID, and significant expressive and receptive language delays in the majority of participants, language appears to be more preserved in individuals with SHANK3 mutations compared to those with $22 \mathrm{q} 13$ deletions seen at the same centers [2, 24]. Motor skills deficits were also pronounced, although early motor milestones were achieved on time for the majority of individuals. Gross motor skills were better developed than fine motor skills and, in most cases, appear to be less severely affected than in individuals with 22q13 deletions, particularly regarding gait. These results indicate that SHANK3 haploinsufficiency affects cognition, language, and motor functioning.

\section{Regression and psychotic symptoms}

Significant cognitive and behavioral regression has been reported in individuals with PMS [2, 3, 9, 51, 55-59].
Over half of our sample reportedly experienced a regression in motor and language skills that occurred during different periods of development (early childhood or adolescence). These results indicate that SHANK3 haploinsufficiency alone is sufficient to increase risk for regression. However, reports of regression must be interpreted with caution based on a lack of well-defined criteria or standardized assessment instruments and potential recall biases in reporting. Further careful study is needed to characterize the regression phenotype in PMS using longitudinal designs and to begin to elucidate the underlying mechanisms.

Possibly related to regression, psychotic symptoms have emerged as an important area of study in PMS as several reports have suggested that as individuals with PMS age, they may be at increased risk for significant psychiatric disturbance, including bipolar disorder [51, 55-57, 59]. Four of the reported patients had truncating mutations in SHANK3 $[9,56]$, indicating that SHANK3 is responsible for this phenotype. Mutations in SHANK3 have also been found in four individuals from two families with atypical schizophrenia associated with early onset and ID [22]. The monozygotic twins reported here (B2, B3) showed "maniclike" behavior beginning at 13 years of age in one and at 9-10 years of age in the other. Also, one individual (S12) experienced psychotic symptoms characterized by auditory and visual hallucinations beginning around 1213 years of age. She had episodic periods of mania and depression, insomnia, decreased appetite and weight loss, unsteady gait, and catatonic posturing, similar to previous reports $[51,55,56,59]$. Importantly, she also had significant regression in language and motor skills with documented cognitive decline from borderline intellectual functioning before puberty to profound ID based on the current assessment at age 42 (see Table 2). The patient was verbally fluent but became non-verbal. She was also walking independently at 20 months and currently is unable to walk more than several steps without support. Pubertal onset appears to be a potential trigger for shifts in the psychiatric phenotype in PMS; hence, it is important to note that only two of the 14 Seaver participants were post-pubertal.

\section{Other medical findings}

Common medical features in individuals with SHANK3 mutations were consistent with published literature in subjects with 22q13.3 deletions [1, 2, 4-6]. Epilepsy has been reported in PMS with a mean prevalence of $32 \%$ and a wide range of seizure types, frequencies, and severity [24]. The lower frequency of seizures in our study compared to that for previously reported individuals with SHANK3 point mutations (29\% versus 57\%) might be due to the young age of many of our patients (seizure onset occurred at $\geq 10$ years in $41 \%$ [7/17] of new and 
previously reported individuals). In agreement with our findings, no specific EEG abnormalities have been reported in PMS, and EEG abnormalities (61\%) are seen in children with and without a history of clinical seizures [24]. Structural brain abnormalities are observed in about a third of cases with 22q13 deletions (including corpus callosum and cerebellar abnormalities, dysmyelination, ventricular dilatation, and arachnoid cysts) $[1,2$, 24]; results from patients with mutations are consistent with those with deletions. Overall, loss of SHANK3 is sufficient to cause seizures and structural brain changes, although findings remain non-specific to PMS.

Gastrointestinal problems, recurrent infections, and increased pain tolerance were common among individuals with SHANK3 mutations, consistent with previous estimates in 22q13 deletions [2, 4]. In agreement with these findings, studies in mice showed that SHANK3 is expressed in the spinal cord and primary sensory neurons, where it regulates pain sensitivity [60]. SHANK3 has also been shown to be expressed in intestinal epithelial cells, where it regulates barrier function [61]. In contrast, despite reports of renal and urinary tract abnormalities in $26-40 \%$ of cases with $22 \mathrm{q} 13$ deletions (including vesicoureteral reflux, hydronephrosis, renal agenesis, and dysplastic or polycystic kidneys) [2, 4], no such anomalies were observed in our cohort. While data from ongoing genotype-phenotype studies are still emerging, it is likely that the genetic risk for renal anomalies is not directly associated to SHANK3 haploinsufficiency and involves other gene(s) in 22q13.

Despite high variability, mild dysmorphic features were prevalent among patients with SHANK3 mutations and were consistent with the phenotype in patients with $22 q 13$ deletions $[1,2,4]$. It has been previously reported that the number of dysmorphic features is correlated with deletion size [2] and that several dysmorphic features are associated with larger deletion sizes [7]. Our results suggest that some of the more common dysmorphic features associated with PMS are caused by SHANK3 mutations, but further studies are needed to determine the contribution of other genes involved in 22q13 deletions.

\section{Conclusions}

This represents a first detailed report of the genetic and phenotypic spectrum associated with SHANK3 mutations, which are being identified with greater frequency as clinical sequencing becomes more widespread. Our findings show that SHANK3 haploinsufficiency due to point mutations alone is sufficient to cause a broad range of phenotypic features associated with PMS. These include hypotonia, global developmental delay, ID, ASD, language deficits, sleep disturbance, increased pain tolerance, regression, motor skills deficits, seizures, abnormal
EEG, brain imaging abnormalities, feeding difficulties, and gastrointestinal problems. We also describe frequent dysmorphic features in individuals with SHANK3 mutations, including fifth finger clinodactyly, long eyelashes, bulbous nose, wide nasal bridge, malocclusion, high arched palate, pointed chin, sandal gap, and dysplastic toenails. Importantly, we show that language and motor phenotypes appear to be less severe in individuals with point mutations, as compared to 22q13 deletions. These findings extend the role of SHANK3 dysfunction in human disease beyond its well-known role at the synapse in the central nervous system.

\section{Additional files}

Additional file 1: Table S1. Loss of function and de novo missense variants in SHANK3 reported previously. Table S2. Clinical features of individuals with pathogenic or likely pathogenic SHANK3 variants reported in the literature. Table S3. In silico prediction of pathogenicity of missense variants in SHANK3 identified in this study and in the literature. Table S4. Reported truncating and in-frame variants in SHANK3 unlikely to be pathogenic. (XLSX 84 kb)

Additional file 2: Table S5. Descriptive and diagnostic data by patient. Table S6. ASD and intellectual ability classifications in individuals with SHANK3 mutations. Table S7. Language and motor functioning in individuals with SHANK3 mutations. (PDF $132 \mathrm{~kb}$ )

\section{Abbreviations \\ ACMG: American College of Medical Genetics and Genomics; ADI-R: Autism Diagnostic Interview-Revised; ADOS-2: Autism Diagnostic Observation Schedule: Second Edition; ASD: Autism spectrum disorder; DAS-II: Differential Ability Scales, Second Edition; DSM-5: Diagnostic and Statistical Manual for Mental Disorders: Fifth Edition; EEG: Electroencephalography; EVS: Exome Variant Server; gnomAD: Genome Aggregation Database; ID: Intellectual disability; IQ: Intellectual quotient; MRI: Magnetic resonance imaging; OMIM: Online Mendelian Inheritance in Man; PMS: Phelan-McDermid syndrome}

\section{Acknowledgements}

We thank the individuals with PMS and their families for their participation.

\section{Funding}

This work was supported by grants from the Beatrice and Samuel A. Seaver Foundation, the Phelan-McDermid Syndrome Foundation, NIMH (R34 MH100276-01 to AK and R21 MH107839 to JDB), and NINDS (U54 NS09209001 to AK and K08 NS091381 to JLH). SDR is a Seaver fellow. JLH is funded by the Robbins Foundation. CB is a Research Director at INSERM. The funding bodies had no role in the design of the study, in the collection, analysis, and interpretation of data, or in the writing of the manuscript.

Availability of data and materials

All data generated or analyzed during this study are included in this article and its supplementary information files.

\section{Authors' contributions}

SDR, PMS, CB, AK, and JDB conceived and designed the study. SDR and CB analyzed and interpreted the genetic data. FM contributed to the molecular validation data and helped with the data processing. PMS, DH, and ATW performed the psychological evaluations. PMS, MPT, and AK analyzed and interpreted the data regarding the psychiatric evaluation. AK, MPT, and CB analyzed and interpreted the data regarding the medical evaluation. YF analyzed and interpreted the data regarding the neurological evaluation. RL analyzed and interpreted the data regarding the dysmorphology exam. AD and JW managed the samples and clinical data. JLH conducted the

phenotypic data collection for individuals B1-B3. AK and JDB supervised the 
study. SDR, PMS, CB, AK, and JDB wrote the manuscript. All authors read and approved the final manuscript.

\section{Ethics approval and consent to participate}

The institutional review boards of the Icahn School of Medicine at Mount Sinai and Baylor College of Medicine approved this study. Participants were enrolled after written informed consent was obtained from parents or legal guardians.

\section{Consent for publication}

Written informed consent for publication was obtained from the parents or legal guardians.

\section{Competing interests}

JDB and Mount Sinai hold a shared parent for the use of insulin-like growth factor-1 (IGF-1) in Phelan-McDermid syndrome. JDB is on the scientific advisory board for Coronis Neuroscience and has consulted for the Gerson Lehrman Group. AK is on the advisory board of Vencerx Therapeutics and consults for Ovid Therapeutics. The remaining authors declare that they have no competing interests.

\section{Publisher's Note}

Springer Nature remains neutral with regard to jurisdictional claims in published maps and institutional affiliations.

\section{Author details}

${ }^{1}$ Seaver Autism Center, Icahn School of Medicine at Mount Sinai, New York, NY 10029, USA. ${ }^{2}$ Department of Psychiatry, Icahn School of Medicine at Mount Sinai, New York, NY 10029, USA. ${ }^{3}$ Department of Neurology, Icahn School of Medicine at Mount Sinai, New York, NY 10029, USA. ${ }^{4}$ Department of Pediatrics, Icahn School of Medicine at Mount Sinai, New York, NY 10029, USA. ${ }^{5}$ Department of Genetics and Genomic Sciences, Icahn School of Medicine at Mount Sinai, New York, NY 10029, USA. ${ }^{6}$ Division of Neurology and Developmental Neuroscience, Department of Pediatrics, Baylor College of Medicine and Texas Children's Hospital, Houston, TX 77030, USA.

${ }^{7}$ Sorbonne Université, INSERM, CNRS, Neuroscience Paris Seine, Institut de Biologie Paris Seine, 75005 Paris, France. ${ }^{8}$ Department of Neuroscience, Icahn School of Medicine at Mount Sinai, New York, NY 10029, USA. ${ }^{9}$ Friedman Brain Institute, Icahn School of Medicine at Mount Sinai, New York, NY 10029, USA. ${ }^{10}$ Mindich Child Health and Development Institute, Icahn School of Medicine at Mount Sinai, New York, NY 10029, USA.

\section{Received: 1 February 2018 Accepted: 13 March 2018}

Published online: 27 April 2018

\section{References}

1. Kolevzon A, Angarita B, Bush L, Wang AT, Frank Y, Yang A, et al. PhelanMcDermid syndrome: a review of the literature and practice parameters for medical assessment and monitoring. J Neurodev Disord. 2014:6:39.

2. Soorya L, Kolevzon A, Zweifach J, Lim T, Dobry Y, Schwartz L, et al. Prospective investigation of autism and genotype-phenotype correlations in 22q13 deletion syndrome and SHANK3 deficiency. Mol Autism. 2013;4:18.

3. Bonaglia MC, Giorda R, Beri S, De Agostini C, Novara F, Fichera M, et al. Molecular mechanisms generating and stabilizing terminal 22q13 deletions in 44 subjects with Phelan/McDermid syndrome. PLoS Genet. 2011;7:e1002173.

4. Sarasua SM, Boccuto L, Sharp JL, Dwivedi A, Chen CF, Rollins JD, et al. Clinical and genomic evaluation of 201 patients with Phelan-McDermid syndrome. Hum Genet. 2014:133:847-59.

5. Luciani JJ, de Mas P, Depetris D, Mignon-Ravix C, Bottani A, Prieur M, et al. Telomeric 22q13 deletions resulting from rings, simple deletions, and translocations: cytogenetic, molecular, and clinical analyses of 32 new observations. J Med Genet. 2003:40:690-6.

6. Wilson HL, Wong AC, Shaw SR, Tse WY, Stapleton GA, Phelan MC, et al. Molecular characterisation of the 22q13 deletion syndrome supports the role of haploinsufficiency of SHANK3/PROSAP2 in the major neurological symptoms. J Med Genet. 2003:40:575-84.

7. Sarasua SM, Dwivedi A, Boccuto L, Rollins JD, Chen CF, Rogers RC, et al. Association between deletion size and important phenotypes expands the genomic region of interest in Phelan-McDermid syndrome (22q13 deletion syndrome). J Med Genet. 2011;48:761-6.
8. Zwanenburg RJ, Ruiter SA, van den Heuvel ER, Flapper BC, Van RavenswaaijArts CM. Developmental phenotype in Phelan-McDermid (22q13.3 deletion) syndrome: a systematic and prospective study in 34 children. J Neurodev Disord. 2016:8:16.

9. Durand CM, Betancur C, Boeckers TM, Bockmann J, Chaste P, Fauchereau F, et al. Mutations in the gene encoding the synaptic scaffolding protein SHANK3 are associated with autism spectrum disorders. Nat Genet. 2007;39:25-7.

10. Moessner R, Marshall CR, Sutcliffe JS, Skaug J, Pinto D, Vincent J, et al. Contribution of SHANK3 mutations to autism spectrum disorder. Am J Hum Genet. 2007;81:1289-97.

11. Gauthier J, Spiegelman D, Piton A, Lafreniere RG, Laurent S, St-Onge J, et al. Novel de novo SHANK3 mutation in autistic patients. Am J Med Genet B Neuropsychiatr Genet. 2009;150B:421-4

12. Boccuto L, Lauri M, Sarasua SM, Skinner CD, Buccella D, Dwivedi A, et al. Prevalence of SHANK3 variants in patients with different subtypes of autism spectrum disorders. Eur J Hum Genet. 2013;21:310-6.

13. Leblond CS, Nava C, Polge A, Gauthier J, Huguet G, Lumbroso S, et al. Metaanalysis of SHANK mutations in autism spectrum disorders: a gradient of severity in cognitive impairments. PLoS Genet. 2014;10:e1004580.

14. De Rubeis S, He X, Goldberg AP, Poultney CS, Samocha K, Cicek AE, et al. Synaptic, transcriptional and chromatin genes disrupted in autism. Nature. 2014:515:209-15.

15. O'Roak BJ, Stessman HA, Boyle EA, Witherspoon KT, Martin B, Lee C, et al. Recurrent de novo mutations implicate novel genes underlying simplex autism risk. Nat Commun. 2014;5:5595.

16. Yuen RK, Merico D, Bookman M, Howe JL. Thiruvahindrapuram B, Patel RV, et al. Whole genome sequencing resource identifies 18 new candidate genes for autism spectrum disorder. Nat Neurosci. 2017;20:602-11.

17. Hamdan FF, Gauthier J, Araki Y, Lin DT, Yoshizawa Y, Higashi K, et al. Excess of de novo deleterious mutations in genes associated with glutamatergic systems in nonsyndromic intellectual disability. Am J Hum Genet. 2011;88:306-16.

18. Gong $X$, Jiang $Y W$, Zhang $X, A n Y, Z$ Zhang J, Wu Y, et al. High proportion of 22q13 deletions and SHANK3 mutations in Chinese patients with intellectual disability. PLoS One. 2012;7:e34739.

19. Redin C, Gerard B, Lauer J, Herenger Y, Muller J, Quartier A, et al. Efficient strategy for the molecular diagnosis of intellectual disability using targeted high-throughput sequencing. J Med Genet. 2014;51:724-36.

20. Lelieveld SH, Reijnders MR, Pfundt R, Yntema HG, Kamsteeg EJ, de Vries P, et al. Meta-analysis of 2,104 trios provides support for 10 new genes for intellectual disability. Nat Neurosci. 2016;19:1194-6.

21. Deciphering Developmental Disorders Study. Prevalence and architecture of de novo mutations in developmental disorders. Nature. 2017;542:433-8.

22. Gauthier J, Champagne N, Lafreniere RG, Xiong L, Spiegelman D, Brustein E, et al. De novo mutations in the gene encoding the synaptic scaffolding protein SHANK3 in patients ascertained for schizophrenia. Proc Natl Acad Sci U S A. 2010;107:7863-8.

23. Betancur C, Buxbaum JD. SHANK3 haploinsufficiency: a "common" but underdiagnosed highly penetrant monogenic cause of autism spectrum disorders. Mol Autism. 2013:4:17.

24. Holder JL Jr, Quach MM. The spectrum of epilepsy and electroencephalographic abnormalities due to SHANK3 loss-of-function mutations. Epilepsia. 2016;57:1651-9.

25. Richards S, Aziz N, Bale S, Bick D, Das S, Gastier-Foster J, et al. Standards and guidelines for the interpretation of sequence variants: a joint consensus recommendation of the American College of Medical Genetics and Genomics and the Association for Molecular Pathology. Genet Med. 2015;17:405-24.

26. Cochoy DM, Kolevzon A, Kajiwara Y, Schoen M, Pascual-Lucas M, Lurie S, et al. Phenotypic and functional analysis of SHANK3 stop mutations identified in individuals with ASD and/or ID. Mol Autism. 2015;6:23.

27. Lord C, Rutter M, DiLavore PS, Risi S, Gotham K, Bishop D. Autism Diagnostic Observation Schedule, 2nd edition (ADOS-2) manual (part I): modules 1-4. Torrance: Western Psychological Services; 2012.

28. Lord C, Rutter M, Le Couteur A. Autism Diagnostic Interview-Revised: a revised version of a diagnostic interview for caregivers of individuals with possible pervasive developmental disorders. J Autism Dev Disord. 1994;24:659-85.

29. American Psychiatric Association. Diagnostic and Statistical Manual of Mental Disorders, 5th edition, text revision. Washington, DC: American Psychiatric Association; 2013.

30. Hus V, Gotham K, Lord C. Standardizing ADOS domain scores: separating severity of social affect and restricted and repetitive behaviors. J Autism Dev Disord. 2014:44:2400-12. 
31. Mullen EM. Mullen Scales of Early Learning. Circle Pines: American Guidance Services; 1995.

32. Roid GH. Stanford Binet Intelligence Scales. 5th ed. Itasca: Riverside Publishing; 2003.

33. Elliot CD. Differential Ability Scales - second edition: introductory and technical manual. San Antonio: Harcourt Assessment; 2007.

34. Bishop SL, Guthrie W, Coffing M, Lord C. Convergent validity of the Mullen Scales of Early Learning and the Differential Ability Scales in children with autism spectrum disorders. Am J Intellect Dev Disabil. 2011;116:331-43.

35. Akshoomoff N. Use of the Mullen Scales of Early Learning for the assessment of young children with autism spectrum disorders. Child Neuropsychol. 2006;12:269-77.

36. Sparrow SS, Cicchetti DV, Balla DA. Vineland Adaptive Behavior Scales: second edition (Vineland II), survey interview form/caregiver rating form. Livonia: Pearson Assessments; 2005.

37. Fenson L, Marchman VA, Thal DJ, Dale PS, Reznick JS, Bates E. MacArthurBates communicative development inventories: user's guide and technical manual. 2nd ed. Baltimore: Brookes Publishing Co.; 2007.

38. Dunn LM, Dunn DM. PPVT-4: Peabody Picture Vocabulary Test. Bloomington: Pearson Assessments; 2007.

39. Williams KT. The Expressive Vocabulary Test. 2nd ed. Circle Pines: AGS Publishing; 2007.

40. Beery KE, Buktenica NA, Beery NA. Beery-Buktenica Developmental Test of Visual-Motor Integration. 6th ed. Minneapolis: Pearson; 2010.

41. Dunn W, Westman K. The sensory profile: the performance of a national sample of children without disabilities. Am J Occup Ther. 1997;51:25-34.

42. Siper PM, Kolevzon A, Wang AT, Buxbaum JD, Tavassoli T. A clinicianadministered observation and corresponding caregiver interview capturing DSM5 sensory reactivity symptoms in children with ASD. Autism Res. 2017;10:1133-40.

43. Nemirovsky SI, Cordoba M, Zaiat JJ, Completa SP, Vega PA, Gonzalez-Moron $D$, et al. Whole genome sequencing reveals a de novo SHANK3 mutation in familial autism spectrum disorder. PLoS One. 2015;10:e0116358.

44. Lim ET, Uddin M, De Rubeis S, Chan Y, Kamumbu AS, Zhang X, et al. Rates, distribution and implications of postzygotic mosaic mutations in autism spectrum disorder. Nat Neurosci. 2017;20:1217-24.

45. Zhang Y, Kong W, Gao Y, Liu X, Gao K, Xie H, et al. Gene mutation analysis in 253 Chinese children with unexplained epilepsy and intellectual/ developmental disabilities. PLoS One. 2015;10:e0141782.

46. Bowling KM, Thompson ML, Amaral MD, Finnila CR, Hiatt SM, Engel KL, et al. Genomic diagnosis for children with intellectual disability and/or developmental delay. Genome Med. 2017;9:43.

47. Samocha KE, Robinson EB, Sanders SJ, Stevens C, Sabo A, McGrath LM, et al. A framework for the interpretation of de novo mutation in human disease. Nat Genet. 2014;46:944-50

48. Bramswig NC, Ludecke HJ, Alanay Y, Albrecht B, Barthelmie A, Boduroglu K, et al. Exome sequencing unravels unexpected differential diagnoses in individuals with the tentative diagnosis of Coffin-Siris and NicolaidesBaraitser syndromes. Hum Genet. 2015;134:553-68.

49. Kolevzon A, Cai G, Soorya L, Takahashi N, Grodberg D, Kajiwara Y, et al. Analysis of a purported SHANK3 mutation in a boy with autism: clinical impact of rare variant research in neurodevelopmental disabilities. Brain Res. 2011;1380:98-105

50. Waga C, Okamoto N, Ondo Y, Fukumura-Kato R, Goto Y, Kohsaka S, et al. Novel variants of the SHANK3 gene in Japanese autistic patients with severe delayed speech development. Psychiatr Genet. 2011;21:208-11.

51. Denayer A, Van Esch $H$, de Ravel T, Frijns JP, Van Buggenhout G, Vogels A et al. Neuropsychopathology in 7 patients with the 22q13 deletion syndrome: presence of bipolar disorder and progressive loss of skills. Mol Syndromol. 2012;3:14-20.

52. Jeffries AR, Curran S, Elmslie F, Sharma A, Wenger S, Hummel M, et al. Molecular and phenotypic characterization of ring chromosome 22. Am J Med Genet A. 2005;137:139-47.

53. Durand CM, Perroy J, Loll F, Perrais D, Fagni L, Bourgeron T, et al. SHANK3 mutations identified in autism lead to modification of dendritic spine morphology via an actin-dependent mechanism. Mol Psychiatry. 2012;17:71-84.

54. Arons MH, Thynne CJ, Grabrucker AM, Li D, Schoen M, Cheyne JE, et al. Autism-associated mutations in ProSAP2/Shank3 impair synaptic transmission and neurexin-neuroligin-mediated transsynaptic signaling. J Neurosci. 2012;32:14966-78.

55. Vucurovic K, Landais E, Delahaigue C, Eutrope J, Schneider A, Leroy C, et al. Bipolar affective disorder and early dementia onset in a male patient with SHANK3 deletion. Eur J Med Genet. 2012;55:625-9.
56. Serret S, Thummler S, Dor E, Vesperini S, Santos A, Askenazy F. Lithium as a rescue therapy for regression and catatonia features in two SHANK3 patients with autism spectrum disorder: case reports. BMC Psychiatry. 2015;15:107.

57. Egger Jl, Zwanenburg RJ, van Ravenswaaij-Arts CM, Kleefstra T, Verhoeven WM. Neuropsychological phenotype and psychopathology in seven adult patients with Phelan-McDermid syndrome: implications for treatment strategy. Genes Brain Behav. 2016;15:395-404.

58. Reierson G, Bernstein J, Froehlich-Santino W, Urban A, Purmann C, Berquist $\mathrm{S}$, et al. Characterizing regression in Phelan McDermid syndrome (22q13 deletion syndrome). J Psychiatr Res. 2017;91:139-44.

59. Verhoeven WM, Egger II, Willemsen MH, de Leijer GJ, Kleefstra T. PhelanMcDermid syndrome in two adult brothers: atypical bipolar disorder as its psychopathological phenotype? Neuropsychiatr Dis Treat. 2012;8:175-9.

60. Han Q, Kim YH, Wang X, Liu D, Zhang ZJ, Bey AL, et al. SHANK3 deficiency impairs heat hyperalgesia and TRPV1 signaling in primary sensory neurons. Neuron. 2016;92:1279-93.

61. Wei SC, Yang-Yen HF, Tsao PN, Weng MT, Tung CC, Yu LCH, et al. SHANK3 regulates intestinal barrier function through modulating ZO-1 expression through the PKCE-dependent pathway. Inflamm Bowel Dis. 2017;23:1730-40.

62. Brandt T, Desai K, Grodberg D, Mehta L, Cohen N, Tryfon A, et al. Complex autism spectrum disorder in a patient with a $17 q 12$ microduplication. Am J Med Genet A. 2012;158A:1170-7.

\section{Submit your next manuscript to BioMed Central and we will help you at every step:}

- We accept pre-submission inquiries

- Our selector tool helps you to find the most relevant journal

- We provide round the clock customer support

- Convenient online submission

- Thorough peer review

- Inclusion in PubMed and all major indexing services

- Maximum visibility for your research

Submit your manuscript at www.biomedcentral.com/submit
Biomed Central 